\title{
Magnetic plasma expulsion
}

\author{
R. E. Phillips and C. A. Ordonez ${ }^{\text {a) }}$ \\ Department of Physics, University of North Texas, Denton, Texas 76203, USA
}

(Received 28 September 2017; accepted 14 December 2017; published online 16 January 2018)

\begin{abstract}
Magnetic confinement of plasmas is widely studied for restricting a plasma's extent to a given region. Presented here is one scheme for what is referred to as magnetic plasma expulsion, which would prevent the plasma from entering a given region. Magnetic plasma expulsion techniques may allow instrumentation access to the interiors of plasmas. Magnetic plasma expulsion techniques may also make alternative approaches for magnetic plasma confinement possible. Particle-in-cell simulation with the Warp code is used to study magnetic plasma expulsion. Some conditions for achieving magnetic plasma expulsion are reported. Published by AIP Publishing. https://doi.org/10.1063/1.5006887
\end{abstract}

\section{INTRODUCTION}

The purpose of magnetic plasma expulsion is to keep plasma from entering a given boundary, in contrast to magnetic plasma confinement, which seeks to contain plasma within a given boundary. The concept of magnetic plasma expulsion presented here is related to work recently reported for control of space charged neutralized ion beams, ${ }^{1}$ though early studies were conducted in a fusion plasma context. ${ }^{2}$ When used in conjunction with magnetic plasma confinement, magnetic plasma expulsion may allow the insertion of instrumentation into a magnetically confined plasma without adversely affecting the confinement properties or purity of the confined plasma. Further, plasma confinement may actually be enhanced via the insertion of field generating electrodes, particle emitters, or particle collectors that control spacecharge effects. There exists a known correlation between the electric potential profile of the interior of certain plasmas and the formation of confinement-enhancing transport barriers. ${ }^{3-5}$

Tokamaks induce a toroidal current within the plasma, eliminating the need for plasma-embedded magnetic coils to achieve a rotational transform. Before the development of tokamaks, however, several confinement schemes were studied that employed multiple current carrying magnetic coils embedded entirely within the plasma. ${ }^{6-8}$ Although these alternative schemes had some highly desirable properties, plasma loss of the support structure of the embedded coils unacceptably deteriorated confinement. Magnetic plasma expulsion may provide an enabling technology for embedding multiple magnetic coils within a plasma, without significant particle loss at the connections to the embedded coils. Early research indicates that when losses to electrical connections and mechanical supports are sufficiently small, classical plasma confinement via certain configurations of multiple plasma-immersed coils may occur. ${ }^{6-8}$ It should also be noted that a Lockheed Martin team is investigating a magnetic fusion reactor concept, which uses multiple magnetic coils embedded within the plasma. ${ }^{9}$ Also, net energy gain is reported as theoretically possible using an inertial

a) Author to whom correspondence should be addressed: cao@unt.edu electrostatic confinement fusion concept that employs magnetic coils embedded within the plasma system. ${ }^{10}$ Both concepts may benefit from reducing plasma loss to a support structure of the embedded coils.

Collaborations such as ALPHA, ATRAP, ASACUSA, AEGIS, and GBAR are involved in or planning for studying antimatter in the form of antihydrogen. ${ }^{1-15}$ Much of the research is focused on comparisons of spectroscopic data between hydrogen and antihydrogen for CPT (charge conjugation, parity, time reversal) symmetry violation studies and on gravity experiments. Production of antihydrogen has been achieved primarily via three-body recombination within plasmas confined by nested Penning traps. One of the difficulties limiting antihydrogen research is the quantity of low speed antihydrogen suitable for experimentation that nested Penning traps can produce, due in part to particle drifts. ${ }^{16}$

New alternatives to nested Penning traps are desirable that can generate low speed antihydrogen in much larger quantities than presently possible. Alternative approaches may also be desirable for achieving electron-positron plasma confinement. The study of such plasmas could enhance the understanding of new phenomena, such as the formation and effects of magnetobound states of positronium. ${ }^{17-19}$ Magnetic plasma expulsion may provide one avenue for the advancement of alternative plasma confinement approaches that employ plasma-embedded magnetic coils. Although levitated multiple plasma-embedded magnetic coil designs have been proposed, ${ }^{20,21}$ physically supporting such coils and providing electrical connections might be accomplished with magnetic plasma expulsion techniques.

By way of example, an alternative plasma confinement concept based on finite-length coaxial solenoids is illustrated in Fig. 1. Using a pair of coaxial solenoids with oppositely directed magnetic fields, the magnetic mirror effect could be used to axially confine a plasma within the inner solenoid. In addition, the coaxial design would have "closed" magnetic field lines that do not cross any material structures at which plasma particles may be lost. Particles in the loss cone of the magnetic mirrors would recirculate back into the inner solenoid by following closed field lines. Such field lines are deflected around the cylindrical material surfaces of metal 


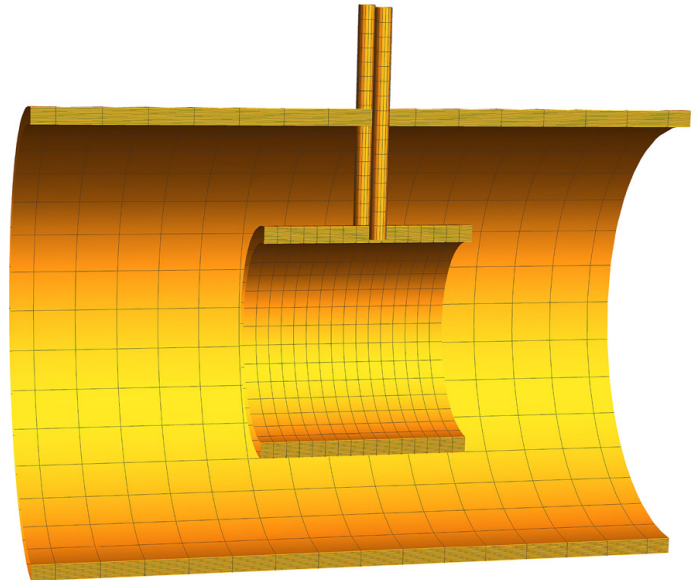

(a)

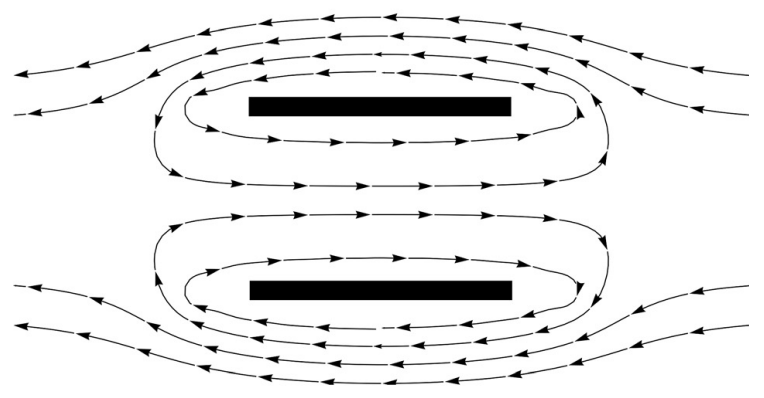

(b)

FIG. 1. Illustration of an alternative plasma confinement approach consisting of two coaxial solenoids. (a) The cylindrical material surfaces of two metal tubes cross the space between the solenoids. These cylindrical material surfaces could enclose mechanical supports and electrical connections to the inner solenoid. (b) The magnetic field produced by the two coaxial solenoids. The outer solenoid is not shown. Plasma would be confined along closed magnetic field lines that enclose the inner solenoid. Two methods would be used to mitigate the loss of plasma particles to the cylindrical material surfaces. Magnetic mirrors would be formed from a field strength gradient along field lines between points within the interior solenoid, where the field is weaker, and points between the solenoids, where the field is stronger. Loss of plasma to the cylindrical material surfaces that cross the space between the two solenoids would also be inhibited by the use of magnetic plasma expulsion to redirect magnetic field lines around the cylindrical material surfaces.

tubes located between the outer and inner solenoids by using a localized magnetic expulsion field as illustrated in Fig. 2. Mechanical supports and electrical connections could pass to the inner solenoid by being located inside of the cylindrical material surfaces.

Section II describes a particle-in-cell simulation used to study magnetic plasma expulsion. Section III describes a parametric study of magnetic plasma expulsion. A discussion and concluding remarks can be found in Sec. IV.

\section{SIMULATION OF MAGNETIC PLASMA EXPULSION}

\section{A. Magnetic field model}

Suppose that one or more cylindrical objects are to be passed through a magnetized plasma in such a way as to

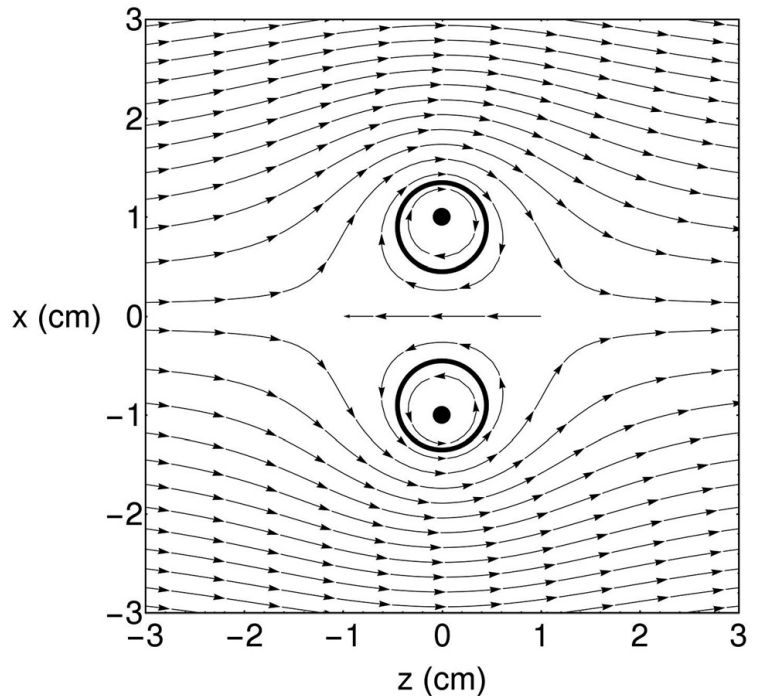

FIG. 2. Illustration of the redirected magnetic field associated with magnetic plasma expulsion used in combination with magnetic plasma confinement. The plasma confinement field is approximated as being uniform near two cylindrical material surfaces (thick solid circles). The expulsion field is approximated as being produced by two straight wires (solid dots) located within the two cylindrical material surfaces. Loss of plasma to the cylindrical material surfaces would be inhibited by the use of magnetic plasma expulsion to redirect plasma along the modified magnetic field.

minimize the effect that their presence has on the plasma's magnetic confinement. For example, cylindrical material surfaces could enclose electrical connections and/or mechanical supports. In the vicinity of the cylindrical material surfaces, assume that the magnetic plasma confinement approach produces an approximately uniform magnetic field. An additional field is to be added to keep the plasma expelled from the region near the cylindrical material surfaces. The magnetic field is then a superposition of a uniform field and whatever additional field is used to achieve magnetic plasma expulsion. For a two-cylinder configuration, the expulsion field is approximated as the field produced by two straight, parallel, infinitesimally thin wires. The combined magnetic field is

$$
\boldsymbol{B}(x, y, z)=B_{m} \boldsymbol{\beta}\left(\frac{x}{S}, \frac{y}{S}, \frac{z}{S}\right)+B_{0} \hat{\boldsymbol{k}} .
$$

Here,

$$
\begin{aligned}
\boldsymbol{\beta}\left(x_{n}, y_{n}, z_{n}\right)= & {\left[-\frac{z_{n}}{\left(x_{n}-1\right)^{2}+z_{n}^{2}}, 0, \frac{\left(x_{n}-1\right)}{\left(x_{n}-1\right)^{2}+z_{n}^{2}}\right] } \\
& -\left[-\frac{z_{n}}{\left(x_{n}+1\right)^{2}+z_{n}^{2}}, 0, \frac{\left(x_{n}+1\right)}{\left(x_{n}+1\right)^{2}+z_{n}^{2}}\right]
\end{aligned}
$$

and $B_{m}=\mu_{0} I /(2 \pi S)$ is the standard expression for the magnetic field strength at a distance $S$ from a single wire, with $I$ the magnitude of current, and $\mu_{0}$ the permeability of free space. Cartesian coordinates are denoted by $x, y, z$, and Cartesian coordinates normalized by $S$ are denoted by $x_{n}, y_{n}$, 
$z_{n}$. The currents carried by the two wires must flow in opposite directions to generate the field shown in Fig. 2. The current in these wires generates an expulsion field characterized by $B_{m}$, which is defined to be the magnitude of the magnetic field at the coordinate system origin generated by a single wire at a distance $S$. A uniform magnetic field approximating the confinement field is superimposed in the $z$ direction parallel to the unit vector $\hat{\mathrm{k}}$ and having magnitude $B_{0}$.

\section{B. Computational model}

A study of plasma behavior is carried out with a Particle-in-Cell (PIC) simulation using the code Warp. ${ }^{2-33}$ Warp has the underlying physics coded into the field solver and particle mover such that it solves for the potential from all particles at predetermined grid points, calculates the electromagnetic fields experienced by each particle, and moves particles according to the Lorentz force law. A guiding center approximation is assumed for the particles during simulation by using an implicit "Drift-Lorentz" mover, which allows time steps larger than the gyro period to be taken. The positional uncertainty as a result of the implicit mover's inability to resolve the gyromotion is less than that introduced by the time step and considered negligible. A detailed description can be found in the literature. ${ }^{23,34}$

With the exception of singularities at the wire locations $x= \pm S, z=0$, the field can be discretized over a numerical mesh grid. A small offset is added to the field description during the discretization to ensure that the wire singularities parallel to the $y$ axis do not overlap the mesh grid. The discretized components of Eq. (1) are

$$
\begin{gathered}
B_{x}=\left(B_{m} S\right)\left[-\frac{k}{(i-S)^{2}+k^{2}+\delta}+\frac{k}{(i+S)^{2}+k^{2}-\delta}\right], \\
B_{y}=0, \\
B_{z}=\left(B_{m} S\right)\left[\frac{i-S}{(i-S)^{2}+k^{2}+\delta}-\frac{i+S}{(i+S)^{2}+k^{2}-\delta}\right]+B_{0} .
\end{gathered}
$$

Here, $i=\left(x_{0} x_{\text {step }}-x_{\text {shift }}\right), k=\left(z_{0} z_{\text {step }}-z_{\text {shift }}\right)$, and $0<x_{0}$ $<x_{\text {grid }}, 0<z_{0}<z_{\text {grid }}$ in integer steps, where $x_{\text {grid }}$ is the number of grid spacings in the $x$ dimension, and $z_{\text {grid }}$ is the number of grid spacings in the $z$ dimension. $x_{\text {shift }}$ and $z_{\text {shift }}$ are added to center the coordinate origin of the simulation inside the simulation volume. $x_{\text {step }}$ and $z_{\text {step }}$ are the grid spacings for a given mesh resolution, and $\delta$ is a slight offset for the wire positions to ensure that a field singularity does not land on a mesh point.

A simulated cubic volume with side $L$ is created with particle absorbing boundary conditions on all faces. The space is divided up with a grid, and the external magnetic field described earlier is passed to Warp in component form on all the grid points. Dirichlet boundary conditions with potential set to zero are used on the boundaries of the simulation at $\pm L / 2$ values of the $x, y$, and $z$ coordinates.

Two cylindrical regions, one centered at each wire that produces the expulsion field, are each defined with a radius $r$ and each represents a cylindrical material surface with a particle absorbing boundary.

\section{Parameter values and base results}

Confinement of an electron-positron plasma is considered. Macroparticles of each species are introduced at starting points equally likely to be anywhere within two source volumes. The two source volumes are located at $\quad\left(-5 \mathrm{~cm}<x_{i}<5 \mathrm{~cm}, \quad-5 \mathrm{~cm}<y_{i}<5 \mathrm{~cm}, \quad-4.8 \mathrm{~cm}<z_{i}\right.$ $<-4.75 \mathrm{~cm})$ and $\left(-5 \mathrm{~cm}<x_{i}<5 \mathrm{~cm},-5 \mathrm{~cm}<y_{i}<5 \mathrm{~cm}\right.$, $\left.4.75 \mathrm{~cm}<z_{i}<4.8 \mathrm{~cm}\right)$, where $x_{i}, y_{i}$, and $z_{i}$ are the starting positions of the created macroparticles. Macroparticles are computational particles that represent a certain number of plasma particles determined by the macroparticle "weight." Since the Lorentz force law depends on the charge to mass ratio, and a macroparticle has the same charge to mass ratio as the plasma particles represented, a macroparticle will have the same trajectory as a single plasma particle. This allows for simulation of large systems of plasma particles in a more computationally efficient manner. Both electron and positron macroparticles are introduced with fully Maxwellian velocity distributions in the $x$ and $y$ dimensions. Half Maxwellian velocity distributions are used in the $z$ dimension, with either a positive or negative $z$ velocity component, such that the macroparticles are initially directed into the simulated volume. The expulsion field generating wires are each displaced a distance $S=1 \mathrm{~cm}$ from the coordinate origin, and the magnetic plasma expulsion field parameter $B_{m}$ is equal to the magnetic plasma confinement field strength $B_{0}$, with $B_{m}=B_{0}=1 \mathrm{~T}$. The $L=10 \mathrm{~cm}$ side cubic simulation volume is divided up with a $x_{\text {grid }} \times y_{\text {grid }} \times z_{\text {grid }}$ $=200 \times 200 \times 200$ grid, such that the grid spacing is $x_{\text {step }}=y_{\text {step }}=z_{\text {step }}=0.5 \mathrm{~mm}$. $x_{\text {shift }}=z_{\text {shift }}=5 \mathrm{~cm}$, and $\delta=1$ $\times 10^{-50}$. The temperature of the electron-positron plasma is $T=40 \mathrm{~K} .400$ macroparticles are inserted into the simulated volume every time step, consisting of 100 electron macroparticles and 100 positron macroparticles created in each of the two source volumes.

Each simulation is run for a time $t_{\max }=90 \mu \mathrm{s}$ divided up into 90000 time steps of length $t_{\text {step }}=1 \mathrm{~ns}$. The total run time is more than 22 times a $10 \mathrm{~cm}$ time-of-flight time based on the thermal speed of the plasma particles. The thermal speed is calculated as $v_{t h}=(k T / m)^{1 / 2}$, where $k$ is Boltzmann's constant, $T$ is the temperature, and $m$ is the mass of a plasma particle. For a magnetic field strength of $1 \mathrm{~T}$, the thermal gyroradius is $(m k T)^{1 / 2} /(e B)=1.4 \times 10^{-5} \mathrm{~cm}$, where $e$ is the elementary charge. The thermal gyroradius is three orders of magnitude smaller than the grid spacing, and the guiding center approximation is considered valid.

A line-averaged number density is used to determine a ratio $\Omega$ of the length $L=10 \mathrm{~cm}$ of the side of the cubic simulation volume to the column Debye length $\lambda_{D}, \Omega=L / \lambda_{D}$. Unless otherwise noted, all values of $\Omega$ are reported at time $t=t_{\max }$. To calculate the column Debye length, the total number of macroparticles is determined in cell columns with dimensions $\Delta x=L / x_{\text {grid }}, \Delta y=L$, and $\Delta z=L / z_{\text {grid }}$. The lineaveraged number density is calculated as 


$$
n=\frac{w N_{\text {positrons }}+w N_{\text {electrons }}}{\Delta V} .
$$

Here, $w=10$ is the macroparticle weight equal to the number of plasma particles per macroparticle, $N_{\text {positrons }}$ is the number of positron macroparticles in the cell column with volume $\Delta V=\Delta x \Delta y \Delta z$, and $N_{\text {electrons }}$ is the number of electron macroparticles in the same cell column. Since the magnetic field has no $y$ dependence, and the guiding center approximation is considered valid, line averaging effectively reduces the spatial distribution of the density to two dimensions. The lineaveraged number density $n$ is used to calculate the column Debye length in one cell column as $\lambda_{D}=\left[\varepsilon_{0} k T /\left(n e^{2}\right)\right]^{1 / 2}$. Here, $\varepsilon_{0}$ is the permittivity of free space. Where $n=0$, the column Debye length is set to $\lambda_{D}=1 \times 10^{12} \mathrm{~cm}$ making $\Omega$ effectively zero. Values $\Omega>1$ are where the column Debye length $\lambda_{D}$ is smaller than the simulated length scale $L$. Values $\Omega>200$ are where the column Debye length is smaller than the grid spacing, making the simulation susceptible to numerical instabilities.

Values of $\Omega$ are used to generate Fig. 3, based on a simulation with the parameter values specified thus far. Figure 3 shows a density-type plot of $\Omega$, with the cylindrical material surfaces at locations chosen for a base case. In the base case, the cylindrical material surfaces have a radius $r=0.5 \mathrm{~cm}$ each and are centered at $x= \pm S, z=0$. The cylindrical material surfaces extend the entire length of the simulated $y$ dimension. Small areas of low density can be seen at the four corners of Fig. 3. These artifacts stem from the magnetic field curvature in the simulation region as seen in Fig. 2. Magnetic field lines near the simulation borders curve into the boundaries of the simulation, which in turn cause artificial losses to particle populations following these field lines. The losses do not affect the plasma behavior near the cylindrical material surfaces. It is possible for macroparticles that are initially created near $x=0$ and that have a sufficiently large velocity component in the $z$ dimension to pass between the cylindrical material surfaces. Such behavior was more

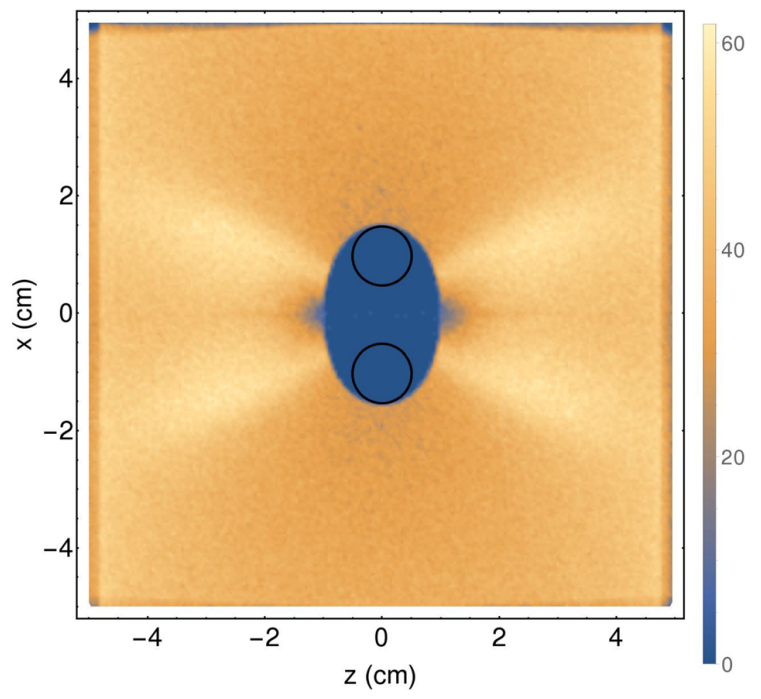

FIG. 3. A density-type plot of $\Omega$ for the base case. The color at a given point represents the value of $\Omega$ at that point. prevalent in a previously reported classical trajectory Monte Carlo study, ${ }^{1}$ when a monoenergtic beam was considered to be incident on a similar magnetic field configuration.

To characterize how effective the expulsion field is, a normalized loss rate $\kappa$ is defined. $\kappa$ is defined as the rate of particle loss to the cylindrical material surfaces with $B_{m}>0$ divided by the rate of particle loss to the cylindrical material surfaces when $B_{m}=0$. Smaller values for $\kappa$ are considered more desirable, indicating that fewer macroparticles are able to cross into the expulsion region. To determine the normalized loss rate $\kappa$, two other parameters are defined. $\chi$ is defined (with $B_{m}>0$ ) as the number of particles lost to the cylindrical material surfaces between times $t_{\max } / 2$ and $t_{\max }$ divided by the number of particles introduced during the same time period. $\chi_{\max }$ is defined as the maximum fraction of particles that would be lost with $B_{m}=0$, and $\chi_{\max }$ is calculated analytically. In the case where no expulsion field is present, $B_{m}=0$, the fraction of all simulated particles lost to the cylindrical material surfaces should approach the fractional area $\chi_{\max }=A_{c m s} / A_{\text {sim }}$ in the limit where $t_{\max }$ tends to infinity and the thermal gyroradius tends to zero. Here, $A_{c m s}=2(2 r L)$ is the cross-sectional area of both volumes enclosed by the cylindrical material surfaces in the $z=0$ plane, and $A_{\text {sim }}=L^{2}$ is the cross-sectional area of a source region. The values $\chi_{\max }=0.1,0.2,0.3,0.4$ are used for values of $r=0.25,0.5,0.75,1.0 \mathrm{~cm}$, assuming that $t_{\text {max }}$ is sufficiently large and that the thermal gyroradius is sufficiently small. The parameter $\kappa$ is calculated as $\kappa=\chi / \chi_{\max }$. For the base case, 14 macroparticles are lost to the cylindrical material surfaces between times $t_{\max } / 2$ and $t_{\max }, 1.8 \times 10^{7}$ macroparticles are injected into the simulation volume during the same time period, $\chi_{\max }=0.2$, and the value of the normalized loss rate is $\kappa=3.9 \times 10^{-6}$.

\section{PARAMETRIC STUDY OF MAGNETIC PLASMA EXPULSION}

\section{A. Limits on parameter value variations}

There are some computational considerations regarding macroparticle weight and plasma temperature. Macroparticle weights that are too large can lead to numerical instabilities, whereas large plasma temperatures can lead to unacceptable positional uncertainty. A series of simulations with variations in macroparticle weight or plasma temperature were run to determine how each parameter individually affected the normalized loss rate $\kappa$ with the magnetic expulsion field turned off, $B_{m}=0$. If $B_{m}$ is set to zero, the value of the normalized loss rate $\kappa$ is expected to be near unity.

The results for the normalized loss rate $\kappa$ versus macroparticle weight variation with $B_{m}=0$ are found in Fig. 4. As the macroparticle weight increased past a critical value, the values of the normalized loss rate $\kappa$ exhibit a decrease attributed to the gradual onset of a numerical instability, which caused some particles to be reflected in the $z$ dimension back toward a source region. Values of the macroparticle weight less than $w=100$ exhibited less than a 15\% deviation from the expected value of unity. For $w=100$, the Debye lengths calculated for a single cell, hereafter referred to as "cell Debye lengths," can reach values smaller than the grid 


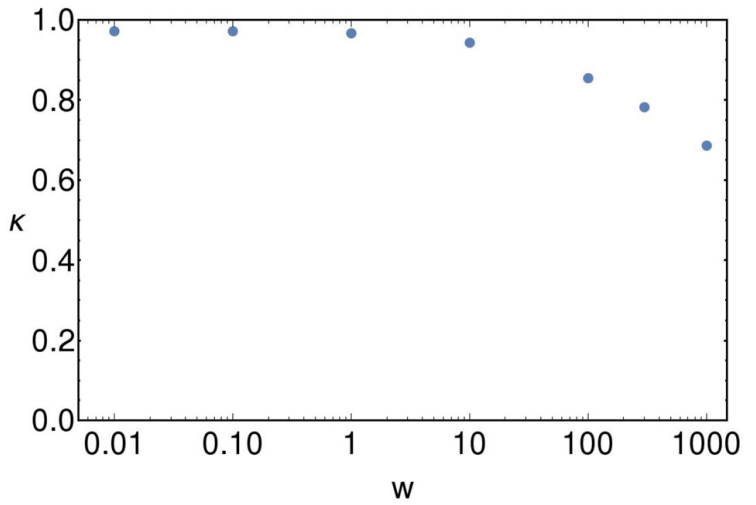

FIG. 4. Normalized loss rate $\kappa$ versus macroparticle weight $w$ with base case parameter values, except with $B_{m}=0$ and with different values for the macroparticle weight $w$.

spacing in some cells. For $w=10$, all cell Debye lengths are larger than the grid spacing. If the cell Debye length is smaller than the grid spacing, numerical instabilities can occur as a result of non-physical interactions. ${ }^{35}$

At sufficiently high plasma temperatures, macroparticles can cross appreciably large sections of the simulated volume in one time step. An erroneously low value for the normalized loss rate $\kappa$ can be reported at high plasma temperatures, because a macroparticle can have a large enough speed to travel through sections of the cylindrical material surfaces in a single time step and not be counted as lost. A plasma temperature study was performed to determine the compatibility of the choice of time step with various plasma temperatures by varying the value of $T$ with $40 \mathrm{~K} \leq T \leq 4.0 \times 10^{7} \mathrm{~K}$, with $B_{m}=0$, and with the macroparticle field solver turned off. Figure 5 shows the resulting normalized loss rate $\kappa$ versus plasma temperature. The field solver is turned off so as to observe particle motion that comes solely from thermal energy and interaction with the external magnetic field configuration. The blue circles are simulated data points, whereas the yellow squares are values predicted by a model. For the model, the guiding center approximation is assumed

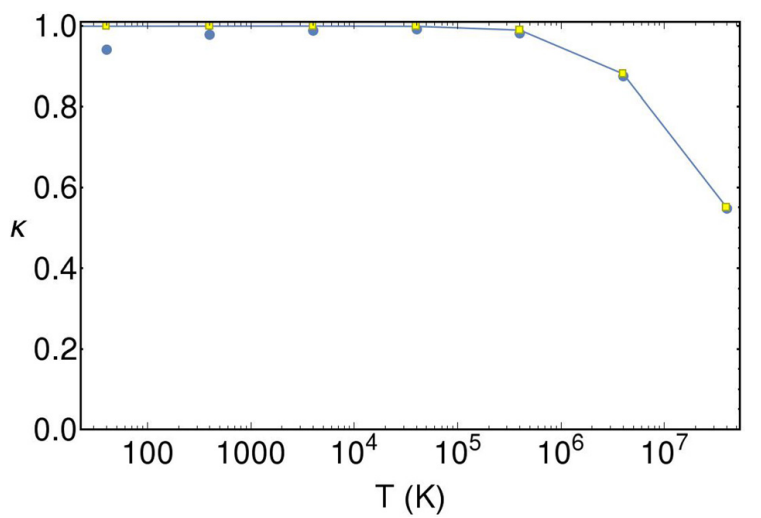

FIG. 5. Normalized loss rate $\kappa$ versus plasma temperature $T$. The simulations are run with base case parameter values, except with $B_{m}=0$, the macroparticle field solver turned off, and the plasma temperature varied. Blue circles are simulated data, while yellow squares are values predicted by an analytical model. valid, such that all particles move only in the $\mathrm{z}$ direction along uniform magnetic field lines with infinitesimally small gyroradii. The model is defined with a coordinate system translation in the $x$ dimension on the range $0<x \leq r$, where $r=0.5 \mathrm{~cm}$ is the radius of a cylindrical material surface region and $x=0$ is the cylindrical material surface center in the $x$ dimension. Since $B_{m}=0$, any particle starting within $0<x \leq r$ will follow a uniform field line, intersect with the cylindrical material surface and should be counted as lost. However, determining if a particle meets the criterion for being lost only occurs at certain times separated by time steps. A spatial step $\delta z=v_{z} t_{\text {step }}$ is defined as the distance a particle moves during a single time step $t_{\text {step }}$ traveling at speed, $v_{z}$. For the model, the normalized loss fraction is $\kappa_{\text {model }}=\chi / \chi_{\max }=P_{\text {detect }}$, where $\chi$ is written as $\chi$ $=P_{\text {detect }} \chi_{\max }$. Here, $P_{\text {detect }}$ is the probability that a particle is detected by the simulation as being lost if the particle is incident on a cylindrical material surface. Particles are equally likely to start anywhere between $0<x \leq r$, such that $f_{d}(x)=1 / r$ is the probability density function describing the particle initial $x$ coordinates. Particles have a half Maxwellian initial velocity distribution, such that a probability density function for particle initial velocity with $0 \leq v_{z}<\infty$ can be written as

$$
f_{v}\left(v_{z}\right)=\sqrt{\frac{2 m}{\pi k T}} e^{-m v_{z}^{2} /(2 k T)} .
$$

With the two probability density functions

$$
P_{\text {detect }}=\int_{0}^{\infty} \int_{0}^{r} P\left(x, v_{z}\right) f_{d}(x) f_{v}\left(v_{z}\right) d x d v_{z},
$$

where

$$
P\left(x, v_{z}\right)= \begin{cases}\frac{c}{\delta z} & \delta z>c \\ 1 & \delta z \leq c\end{cases}
$$

is the probability for a particle with initial coordinate $\left(x, v_{z}\right)$ to be detected, and $c=2 \sqrt{r^{2}-x^{2}}$ is the chord length across the cylindrical material surface at coordinate $x$. Numerical integration yields the model in Fig. 5.

By taking $B_{m}=0$, and with the macroparticle field solver turned off, the expected normalized loss rate approaches one, $\kappa \rightarrow 1.0$, as $t_{\max } \rightarrow \infty$. A significant deviation from the expected normalized loss rate $\kappa=1.0$ is attributed to the positional uncertainty inherent at higher plasma temperatures. As $T$ increases, those temperatures that recover within $15 \%$ of the expected normalized loss rate $(\kappa \geq 0.85)$ are considered to be within acceptable limits for predicting particle loss to the cylindrical material surfaces. Those plasma temperatures that show a larger deviation from the expected loss rate $(\kappa<0.85)$ in Fig. 5 would require a smaller time step to more accurately simulate magnetic plasma expulsion. Temperatures below $T=4.0 \times 10^{6} \mathrm{~K}$ are considered to be within acceptable limits (see Fig. 5).

\section{B. Debye length}

A change in the column Debye length of the plasma can be accomplished by varying the plasma temperature or the 
macroparticle weight. The effect of plasma temperature and macroparticle weight variation on the normalized loss rate $\kappa$ is evaluated using a parameter $\Omega_{p}$, which is intended to represent the value of $\Omega$ in the undisturbed plasma. $\Omega_{p}$ is defined to have the value of $\Omega$ at a point away from the distortion at $x=4.0 \mathrm{~cm}, z=4.0 \mathrm{~cm}$. Figure 6 shows the results. The maximum value of $\kappa$ in Fig. 6 is $\kappa=7.25 \times 10^{-5}$. For the value of $\Omega_{p}>100, \kappa$ is zero. The simulated data are taken using base case values, other than the parameter that is varied. For the plasma temperature variations, a macroparticle weight of $w=10$ is used, while for the macroparticle weight variations, a plasma temperature of $T=40 \mathrm{~K}$ is used. In the base case, the value of $\Omega_{p}$ is $\Omega_{p}=46.05$, which, for $T=40 \mathrm{~K}$, corresponds to a line-averaged density of $4.04 \times 10^{10} \mathrm{~m}^{-3}$.

\section{Magnetic plasma expulsion field and cylindrical material surface radius}

A parameter scan is performed over $0.5 \mathrm{~T} \leq B_{m} \leq 4.0 \mathrm{~T}$ in increments of $0.5 \mathrm{~T}$, and over $0.25 \mathrm{~cm} \leq r \leq 1.0 \mathrm{~cm}$ in increments of $0.25 \mathrm{~cm}$, with other parameters having base case values. Figure 7 shows values of the normalized loss rate $\kappa$ for the permutations of $r$ and $B_{m} . \kappa$ varies from a maximum value of $\kappa=0.059$ for $r=1.0 \mathrm{~cm}$ and $B_{m}=0.5 \mathrm{~T}$, to a minimum value of $\kappa=1.11 \times 10^{-6}$ for $r=0.25 \mathrm{~cm}$ and $B_{m}=2.5 \mathrm{~T}$. For a given value of $r$, the value of $\kappa$ is larger than 0.01 for $B_{m}$ less than a threshold value, and the value of $\kappa$ is smaller than $10^{-5}$ for $B_{m}$ greater than the threshold value. Also, the threshold value of $B_{m}$ increases with $r$.

\section{Magnetic plasma confinement field}

Figure 8 shows the results of a variation of the uniform field strength $B_{0}$. Despite being an implicit particle mover, the drift-Lorentz mover in Warp retains information about the gyroradius. When the gyroradius sizes are sufficiently large, the values for the normalized loss rate $\kappa$ can be affected. The gyroradius of a charged particle is $r_{g}=\left(m v_{\perp}\right) /(|q| B)$, where $m$ is the mass, $|q|$ is the absolute

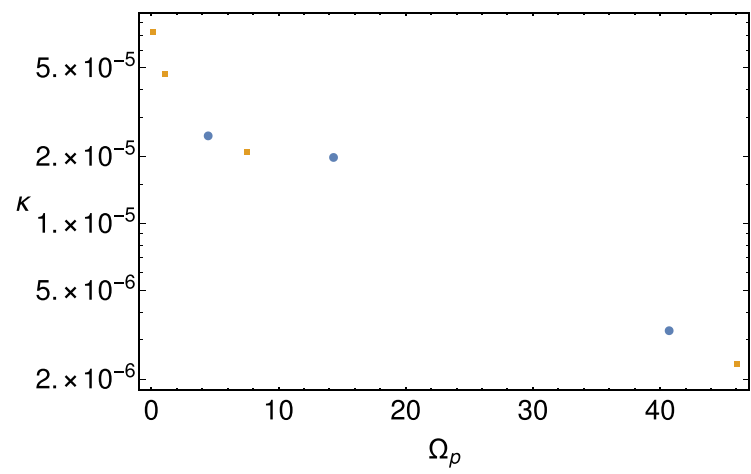

FIG. 6. Normalized loss rate $\kappa$ versus $\Omega_{p}$. For variations of macroparticle weight (blue circular dots), the temperature is $T=40 \mathrm{~K}$, and the values are $w=0.1\left(\Omega_{p}=4.49\right), w=1.0\left(\Omega_{p}=14.35\right), w=10\left(\Omega_{p}=40.73\right), w=100$ $\left(\Omega_{p}=108.44\right)$. For variations of plasma temperature (yellow squares), the macroparticle weight is $w=10$, and the values are $T=40 \mathrm{~K}\left(\Omega_{p}=46.05\right)$, $T=400 \mathrm{~K}\left(\Omega_{p}=7.53\right), T=4000 \mathrm{~K}\left(\Omega_{p}=1.12\right), T=40000 \mathrm{~K}\left(\Omega_{p}=0.14\right)$. Otherwise, base case parameter values are used. The value of $\kappa$ is zero for $\Omega_{p}=108.44$, and the point is not shown

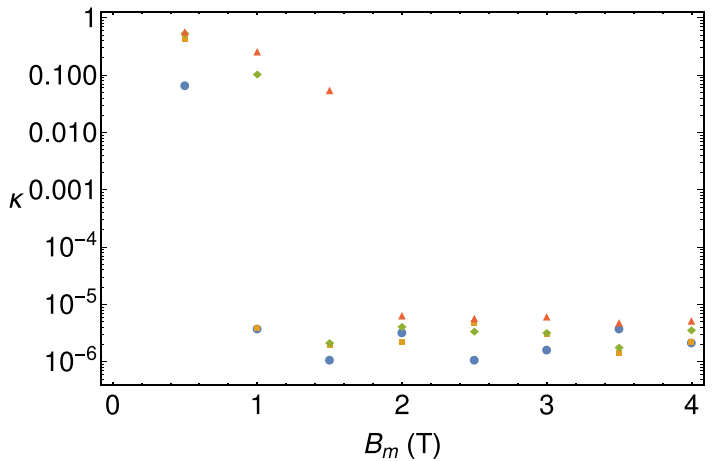

FIG. 7. Normalized loss rate $\kappa$ versus magnetic plasma expulsion field parameter $B_{m}$ for $r=0.25 \mathrm{~cm}$ (circles), $r=0.5 \mathrm{~cm}$ (squares), $r=0.75 \mathrm{~cm}$ (diamonds), and $r=1.0 \mathrm{~cm}$ (triangles).

value of charge, $B$ is the magnitude of the magnetic field, and $v_{\perp}$ is the particle's velocity component perpendicular to the magnetic field. Due to a non-negligible gyroradius, macroparticles that would otherwise follow magnetic field lines around the distortion can be lost to the cylindrical material surfaces causing an increase in the normalized loss rate. The increase in $\kappa$ in Fig. 8 is attributed to the effect of a finite gyroradius size.

\section{E. Charge separation}

A phenomenon that should be considered is that of charge separation. Charge separation may occur as plasma particles travel around the expulsion region due to a sign dependent cross-magnetic-field drift experienced by the plasma particles. The resulting non-neutrality causes an electric field $\boldsymbol{E}$ to form. To assess the effect, a simulation is run with base case parameter values except for a variation of the uniform magnetic field strength, $5.0 \times 10^{-6} \mathrm{~T} \leq B_{0} \leq 0.5 \mathrm{~T}$. Due to the symmetry of the simulation about the $x=0$ plane, the $y$ component of the electric field on only one side of the expulsion region, along $1.5 \mathrm{~cm} \leq x \leq 5 \mathrm{~cm}, y=0, z=0$ is recorded. The maximum value of the electric field at $t=t_{\max }$ is used to estimate the maximum $\boldsymbol{E} \times \boldsymbol{B}$ drift speed of the particles as $v_{\text {drift }}=|\boldsymbol{E} \times \boldsymbol{B}| / B^{2}$. Figure 9 shows the results of the study. For magnetic field values near the base case value, the maximum drift speed is many orders of magnitude below the thermal speed.

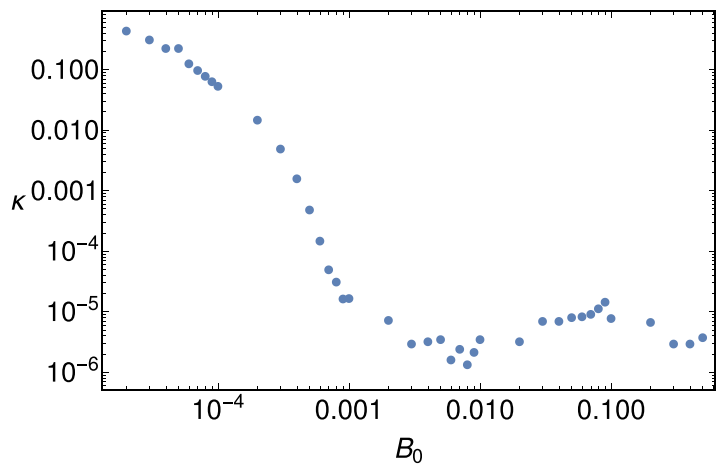

FIG. 8. Normalized loss rate $\kappa$ versus uniform magnetic field strength $B_{0}$, with base case values otherwise. 


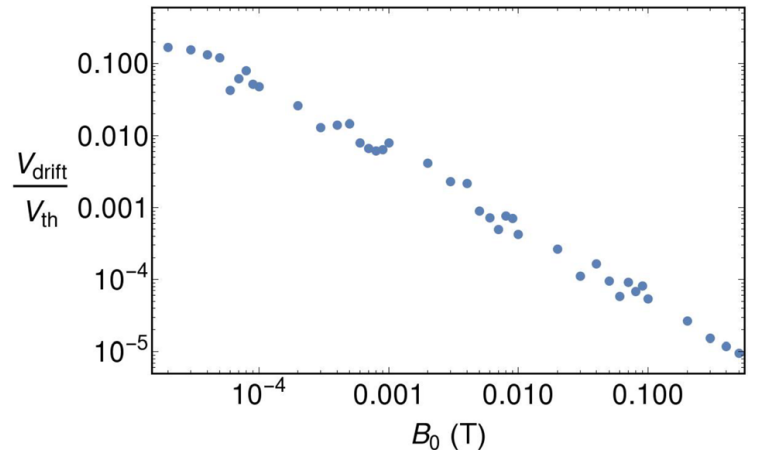

FIG. 9. Maximum $\boldsymbol{E} \times \boldsymbol{B}$ drift speed normalized by the thermal speed at $S+r \leq x \leq L / 2, y=0, z=0$. All parameter values are the same as the base case, except for the value of $B_{0}$.

\section{F. Particle mass}

An electron-positron plasma has been considered up to this point, with all particles having the same mass. The effect of changing the particle mass is evaluated by also considering a proton-antiproton plasma. Two simulations are compared. One simulation is the base case electron-positron plasma. The other simulation is a proton-antiproton plasma with base case parameter values, except that the time step is increased by a factor of the square root of the mass ratio, $t_{\text {step }}=42.85 \mathrm{~ns}$. With such a change, equal numbers of particles are introduced in the two simulations, and the particles travel the same average distance during a time step.

For the electron-positron base-case simulation, 14 macroparticles are lost to the cylindrical material surfaces between times $t_{\max } / 2$ and $t_{\max }, 1.8 \times 10^{7}$ macroparticles are injected into the simulation volume during the same time period, $\chi_{\max }=0.2$, and the value of the normalized loss rate is $\kappa=3.9 \times 10^{-6}$. For the proton-antiproton plasma simulation, 16 macroparticles are lost to the cylindrical material surfaces between times $t_{\max } / 2$ and $t_{\max }, 1.8 \times 10^{7}$ macroparticles are injected into the simulation volume during the same time period, $\chi_{\max }=0.2$, and the value of the normalized loss rate is $\kappa=4.4 \times 10^{-6}$. Figure 10 shows a density plot of the proton-antiproton plasma. The magnetic plasma expulsion behavior is nearly the same as in Fig. 3 for an electron-positron plasma. However, there is a noticeable stream of particles traversing the gap between the two cylindrical material surfaces, indicating that plasma particles with greater mass may more readily pass between the cylindrical material surfaces.

Two more simulations are compared with the same parameter values as the preceding two, except with the value $r=0.25 \mathrm{~cm}$. Three (3) macroparticles are lost to the cylindrical material surfaces in both the electron-positron plasma simulation and the proton-antiproton plasma simulation, corresponding to $\kappa=1.6 \times 10^{-6}$ for each simulation.

\section{DISCUSSION AND CONCLUSION}

In the past, various alternative magnetic plasma confinement approaches have been proposed that would require magnetic-field-producing coils to be located inside of

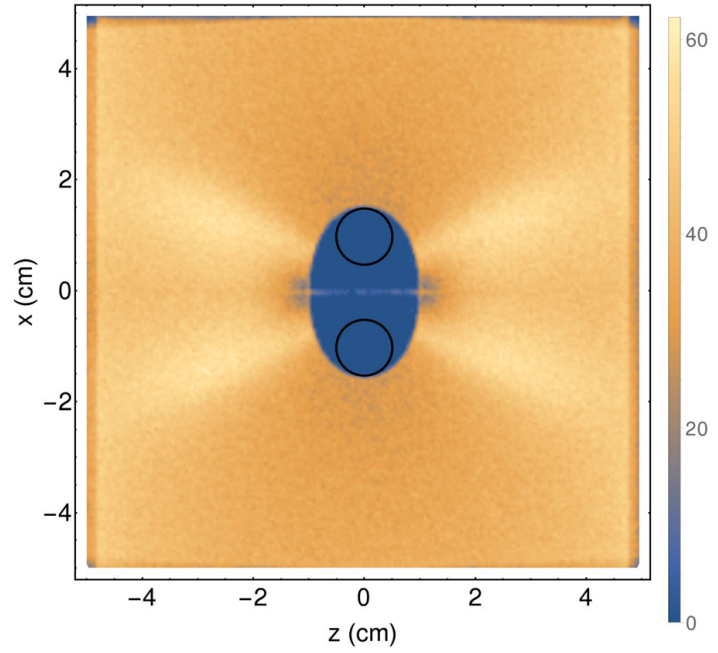

FIG. 10. Same as Fig. 3, except for a proton-antiproton plasma, with an increased time step.

confined plasma. The development of magnetic plasma expulsion techniques may enable mechanical supports and electrical connections to pass through the confined plasma to such coils, without adversely affecting the properties of the confined plasma. Magnetic plasma expulsion would keep plasma away from the mechanical supports and electrical connections by redirecting the local magnetic field around such structures.

By way of example, an alternative plasma confinement approach that makes use of magnetic plasma expulsion was described (see Fig. 1). The approach would use two coaxial solenoids, with one solenoid located inside of the other solenoid, and with a pair of metal tubes passing between the two solenoids. The pair of metal tubes could contain mechanical supports and electrical connections. There would be magnetic field lines that pass through the inner solenoid and that would also pass through the metal tubes, were it not for the use of magnetic plasma expulsion. Instead, such magnetic field lines become closed field lines, which do not intersect the metal tubes. Plasma particles located within the inner solenoid that follow such field lines may return to the region within the inner solenoid, where the magnetic field strength is weakest. Plasma confined within the inner solenoid is describable as being confined by a magnetic mirror with closed field lines. It is also noted that it is possible for plasma particles to pass between the metal tubes. Furthermore, it may be possible to locate particle sources between the metal tubes, for injecting particles into the confined plasma. Such injection of particles could serve as a means to help control the density, temperature, and/or neutrality of the plasma.

Particle-in-cell simulation of a model system was used to investigate the concept of magnetic plasma expulsion. The model system consisted of a magnetically confined electronpositron plasma in the vicinity of a pair of metal tubes that pass through the plasma. Without magnetic plasma expulsion, plasma particles could follow field lines that pass through the metal tubes and be lost. A figure of merit was defined as the ratio of the loss rate to the metal tubes with 
magnetic plasma expulsion present to the loss rate to the metal tubes without a magnetic plasma expulsion present. Values for the figure of merit were found to be much less than one for a variety of parameter values. It is concluded that magnetic plasma expulsion may serve to reduce the adverse effects associated with passing a material object through an electron-positron plasma and possibly other plasmas with parameter values similar to those considered here.

\section{ACKNOWLEDGMENTS}

Computational resources were provided by UNT's High Performance Computing Services, a division of the University Information Technology with additional support from UNT Office of Research and Economic Development. This material is based upon work supported by the National Science Foundation under Grant No. PHY-1500427 and by the Department of Energy under Grant No. DE-FG02-06ER54883. The authors would like to thank David Grote, Jean-Luc Vay, Ryan Lane, and Ryan Hedlof for helpful discussions.

${ }^{1}$ R. E. Phillips and C. A. Ordonez, Phys. Proc. 66, 148 (2015).

${ }^{2}$ B. Lehnert, Plasma Phys. 17, 501 (1975).

${ }^{3}$ K. H. Burrell, Phys. Plasmas 4, 1499 (1997)

${ }^{4}$ E. J. Synakowski, Plasma Phys. Controlled Fusion 40, 581 (1998).

${ }^{5}$ E. J. Doyle, W. A. Houlberg, Y. Kamada, V. Mukhovatov, T. H. Osborne, A. Polevoi, G. Bateman, J. W. Connor, J. G. Cordey, T. Fujita et al., Nucl. Fusion 47, S18 (2007)

${ }^{6}$ S. Yoshikawa, Nucl. Fusion 13, 433 (1973)

${ }^{7}$ J. C. Sprott and S. C. Prager, Nucl. Fusion 25, 1179 (1985).

${ }^{8}$ M. W. Maisel, T. Ohkawa, K. H. Burrell, R. L. Freeman, F. J. Helton, T. H. Jensen, R. J. La Haye, D. O. Overskei, R. Prater, J. M. Rawls, and T. Tamano, Nucl. Fusion 25, 1113 (1985).

${ }^{9}$ T. McGuire, Bull. Am. Phys. Soc. 61, DPP.NP10.180 (2016).

${ }^{10}$ J. Hedditch, R. Bowden-Reid, and J. Khachan, Phys. Plasmas 22, 102705 (2015).

${ }^{11}$ M. Ahmadi, B. X. R. Alves, C. J. Baker, W. Bertsche, E. Butler, A. Capra, C. Carruth, C. L. Cesar, M. Charlton, S. Cohen, R. Collister, S. Eriksson, A. Evans, N. Evetts, J. Fajans, T. Friesen, M. C. Fujiwara, D. R. Gill, A. Gutierrez, J. S. Hangst, W. N. Hardy, M. E. Hayden, C. A. Isaac, A. Ishida, M. A. Johnson, S. A. Jones, S. Jonsell, L. Kurchaninov, N. Madsen, M. Mathers, D. Maxwell, J. T. K. McKenna, S. Menary, J. M. Michan, T. Momose, J. J. Munich, P. Nolan, K. Olchanski, A. Olin, P. Pusa, C. O. Rasmussen, F. Robicheaux, R. L. Sacramento, M. Sameed, E. Sarid, D. M. Silveira, S. Stracka, G. Stutter, C. So, T. D. Tharp, J. E.
Thompson, R. I. Thompson, D. P. van der Werf, and J. S. Wurtele, Nat Commun. 8, 681 (2017).

${ }^{12}$ G. Gabrielse, R. Kalra, W. S. Kolthammer, R. McConnell, P. Richerme, D. Grzonka, W. Oelert, T. Sefzick, M. Zielinski, D. W. Fitzakerley, M. C. George, E. A. Hessels, C. H. Storry, M. Weel, A. Müllers, and J. Walz, Phys. Rev. Lett. 108, 113002 (2012).

${ }^{13}$ N. Kuroda, S. Ulmer, D. J. Murtagh, S. Van Gorp, Y. Nagata, M. Diermaier, S. Federmann, M. Leali, C. Malbrunot, V. Mascagna, O. Massiczek, K. Michishio, T. Mizutani, A. Mohri, H. Nagahama, M. Ohtsuka, B. Radics, S. Sakurai, C. Sauerzopf, K. Suzuki, M. Tajima, H. A. Torii, L. Venturelli, B. Wunschek, J. Zmeskal, N. Zurlo, H. Higaki, Y. Kanai, E. Lodi Rizzini, Y. Nagashima, Y. Matsuda, E. Widmann, and Y. Yamazaki, Nat. Commun. 5, 3089 (2014).

${ }^{14}$ P. Scampoli and J. Storey, Mod. Phys. Lett. A 29, 1430017 (2014).

${ }^{15}$ P. Perez, D. Banerjee, F. Biraben, D. Brook-Roberge, M. Charlton, P. Clade, P. Comini, P. Crivelli, O. Dalkarov, P. Debu et al., Hyperfine Interact. 233, 21 (2015).

${ }^{16}$ C. A. Ordonez and D. L. Weathers, Phys. Plasmas 15, 083504 (2008).

${ }^{17}$ J. R. Correa and C. A. Ordonez, Phys. Plasmas 21, 082115 (2014).

${ }^{18}$ F. F. Aguirre and C. A. Ordonez, Phys. Rev. E 91, 033103 (2015).

${ }^{19}$ F. F. Aguirre and C. A. Ordonez, Phys. Plasmas 24, 104502 (2017).

${ }^{20}$ J. D. Wofford and C. A. Ordonez, AIP Conf. Proc. 1525, 106 (2013).

${ }^{21}$ R. A. Lane and C. A. Ordonez, AIP Adv. 4, 077117 (2014).

${ }^{22}$ A. Friedman, R. H. Cohen, D. P. Grote, S. M. Lund, W. M. Sharp, J.-L.

Vay, I. Haber, and R. A. Kishek, IEEE Trans. Plasma Sci. 42, 1321 (2014).

${ }^{23}$ J.-L. Vay, D. P. Grote, R. H. Cohen, and A. Friedman, Comput. Sci. Discovery 5, 014019 (2012).

${ }^{24}$ J.-L. Vay, C. G. R. Geddes, E. Cormier-Michel, and D. P. Grote, J. Comput. Phys. 230, 5908 (2011).

${ }^{25}$ S. M. Lund, T. Kikuchi, and R. C. Davidson, Phys. Rev. Spec. Top. Accel. Beams 12, 114801 (2009).

${ }^{26}$ J.-L. Vay, Phys. Rev. Lett. 98, 130405 (2007).

${ }^{27}$ S. M. Lund, S. H. Chilton, and E. P. Lee, Phys. Rev. Spec. Top. -Accel. Beams 9, 064201 (2006)

${ }^{28}$ J.-L. Vay, P. Colella, J. W. Kwan, P. McCorquodale, D. B. Serafini, A. Friedman, D. P. Grote, G. Westenskow, J.-C. Adam, A. Héron, and I. Haber, Phys. Plasmas 11, 2928 (2004).

${ }^{29}$ D. P. Grote, A. Friedman, J.-L. Vay, and I. Haber, AIP Conf. Proc. 749, 55 (2005).

${ }^{30}$ A. Friedman, D. P. Grote, and I. Haber, Phys. Fluids B: Plasma Phys. 4, 2203 (1992)

${ }^{31}$ K. Gomberoff, J. Fajans, A. Friedman, D. Grote, J.-L. Vay, and J. S. Wurtele, Phys. Plasmas 14, 102111 (2007).

${ }^{32}$ K. Gomberoff, J. Fajans, J. Wurtele, A. Friedman, D. P. Grote, R. H. Cohen, and J.-L. Vay, Phys. Plasmas 14, 052107 (2007).

${ }^{33}$ A. Narimannezhad, C. J. Baker, M. H. Weber, J. Jennings, and K. G. Lynn, Eur. Phys. J. D 68, 351 (2014).

${ }^{34}$ R. H. Cohen, A. Friedman, D. P. Grote, and J.-L. Vay, Nucl. Instrum. Methods Phys. Res., A 577, 52 (2007).

${ }^{35}$ L. Garrigues, G. Fubiani, and J. P. Boeuf, J. Appl. Phys. 120, 213303 (2016). 